\title{
Self-Limiting Size Distribution of Supported Cobalt Nanoclusters at Room Temperature
}

\author{
Shangjr Gwo, ${ }^{1, *}$ Chung-Pin Chou, ${ }^{1}$ Chung-Lin Wu, ${ }^{1}$ Yi-Jen Ye, ${ }^{1}$ Shu-Ju Tsai, ${ }^{1}$ Wen-Chin Lin,${ }^{2}$ and Minn-Tsong Lin ${ }^{2, \dagger}$ \\ ${ }^{1}$ Department of Physics, National Tsing-Hua University, Hsinchu 300, Taiwan, Republic of China \\ ${ }^{2}$ Department of Physics, National Taiwan University, Taipei 106, Taiwan, Republic of China \\ (Received 28 August 2002; revised manuscript received 20 February 2003; published 9 May 2003; \\ publisher error corrected 13 June 2003)
}

\begin{abstract}
Formation of monodispersed Co nanoclusters on a single-crystal $\mathrm{Si}_{3} \mathrm{~N}_{4}$ dielectric film at room temperature is reported. A remarkably narrow size distribution with the average size of $\sim 30$ Co atoms has been obtained. We have confirmed that the average size of Co nanoclusters is independent of the Co coverage and the cluster areal density linearly proportions to the Co deposition amount even at high coverages. Also, we have found that Co nanoclusters deposited on $\mathrm{Si}_{3} \mathrm{~N}_{4}$ are thermally stable with respect to cluster aggregation/coalescence. We propose that this novel phenomenon is a quantum size effect, manifested by local energy minima in the electronic shell structure of Co quantum dots.
\end{abstract}

DOI: 10.1103/PhysRevLett.90.185506

Unusual physical and chemical properties of nanoclusters depend strongly on the cluster size. Nanocluster properties such as the specific heat and the magnetic susceptibility can alter drastically even when the cluster size varied by a single atom $[1,2]$. For transition metal clusters supported on insulating films, which are widely studied as model heterogeneous catalysts, it has been found that the size of clusters is very important for their catalytic activity and selectivity in the ultrasmall size regime ( $<100$ atoms) [2,3]. And, for the magnetic nanoclusters such as $\mathrm{Fe}, \mathrm{Co}, \mathrm{Ni}$, their magnetism at ultrasmall length scales has a wealth of scientific interest and technological importance [2]. Therefore, when utilizing or studying the size-dependent properties, it is very crucial to be able to prepare monodispersed clusters on well-characterized supporting surfaces. For metallic clusters prepared in the free space, both electronic shell structures and structural stability can play important roles in determining magic-sized clusters with preferred numbers of atoms or structures [4]. However, it is more difficult to control the size of nanoclusters supported on a substrate because of the complications involved with the interactions between clusters and the substrate and the kinetic processes of atoms nucleated and grown on the substrate.

In this Letter, we report the use of single-crystal $\mathrm{Si}_{3} \mathrm{~N}_{4}$ film as the support for forming monodispersed Co nanoclusters. The motivation for using a single-crystal $\mathrm{Si}_{3} \mathrm{~N}_{4}$ support is twofold. First, the dielectric support reduces chemical intermixing (such as the formation of silicides on a $\mathrm{Si}$ substrate) and electronic coupling $\left(\mathrm{Si}_{3} \mathrm{~N}_{4}\right.$ is an excellent diffusion barrier with a bandgap energy of $\sim 4-5 \mathrm{eV}$ ) between metal clusters and the substrate compared with situations using semiconductor or metal supporting surfaces. Second, the defect-free $\mathrm{Si}_{3} \mathrm{~N}_{4}$ surface provides us a unique opportunity to study the formation of metal clusters without the influence of surface defects. Consequently, quantum effect can play an important role in the size control.
PACS numbers: 61.46.+w, 36.40.Qv, 68.35.Md, 68.55.Ac

The experiments were performed in an ultrahigh vacuum (base pressure $<1 \times 10^{-10}$ Torr) system. Single-crystal $\beta$ - $\mathrm{Si}_{3} \mathrm{~N}_{4}(0001)-(4 \times 4)$ [the basal plane lattice constant $a_{\beta-\mathrm{Si}_{3} \mathrm{~N}_{4}}=7.61 \AA$; the $2 \times 2$ cell of the $\operatorname{Si}(111)$ substrate $\left(a_{\mathrm{Si}}=3.84 \AA\right)$ is only $\sim 1 \%$ bigger than the unit cell of $\left.\beta-\mathrm{Si}_{3} \mathrm{~N}_{4}(0001)\right]$ surface was prepared on boron-doped $p$-silicon (111) substrates by thermal nitridation with an $\mathrm{NH}_{3}$ gas at $1200 \mathrm{~K}$. The thickness of the nitride film determined by high-resolution crosssectional transmission electron microscopy is $\sim 1.2 \mathrm{~nm}$. The details about the sample nitridation process can be found elsewhere [5,6]. After the nitridation procedure, the nitridated $\mathrm{Si}$ sample was quenched to room temperature and dosed with an electron-beam evaporator. The deposition rate ranged from 0.3 to $1.2 \mathrm{ML} / \mathrm{min}$ [1 ML is equivalent to the nominal surface atom density of hcp $\mathrm{Co}(0001)$ or fcc $\mathrm{Co}(111)$, i.e., $1 \mathrm{ML}=1.83 \times$ $10^{15}$ atoms $\left./ \mathrm{cm}^{2}\right]$. The sample imaging bias was around $-4.2 \mathrm{~V}$, which corresponds to a dominant surface filled state on the $\mathrm{Si}_{3} \mathrm{~N}_{4}$ film $[5,6]$. We obtained the size distributions of deposited Co clusters using an image analysis program (Scion Image). To reduce the tip-size effect, we only analyzed results taken with very sharp tips, in which clear atomic-resolution images on the reconstruction structure of the $\mathrm{Si}_{3} \mathrm{~N}_{4}$ support can be simultaneously obtained.

High-resolution STM images were used to determine the diameter, height, shape, and density of the Co clusters. Figure 1 shows the STM images of Co nanoclusters grown on the $\beta-\mathrm{Si}_{3} \mathrm{~N}_{4}(0001)-(4 \times 4)$ surface at three different adatom coverages $(0.10,0.17$, and $0.36 \mathrm{ML}$ of Co) and the corresponding histograms of cluster diameter and height. The room-temperature deposited Co nanoclusters display a striking feature of self-limiting size distribution at different adatom coverages: The mean cluster diameter $(\langle d\rangle)$ is $\sim 1.40-1.55 \mathrm{~nm}$; the cluster height $(h)$ distributes between 1 to $3 \mathrm{ML}$ height of Co; and the diameter-distribution width $\sigma\left[\left(\left\langle d^{2}\right\rangle-\langle d\rangle^{2}\right)^{1 / 2}\right]$ for all three coverages is $\sim 0.35-0.40 \mathrm{~nm}$. Also, we did 

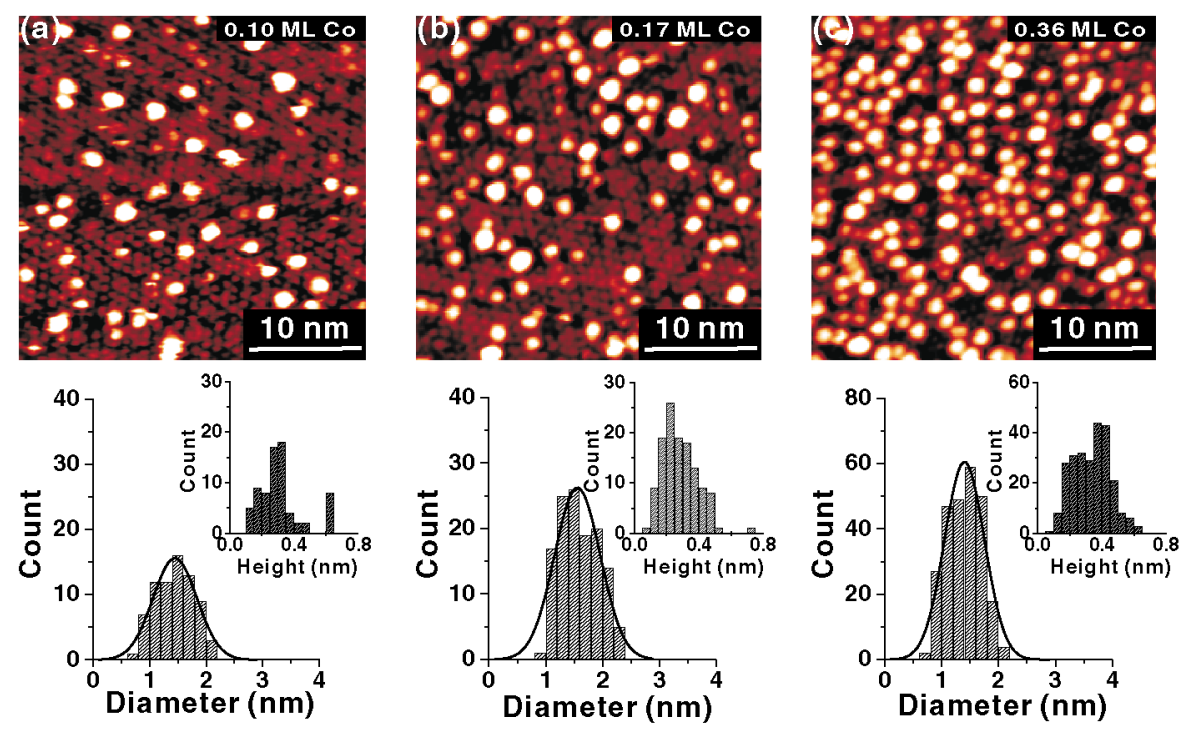

FIG. 1 (color). (a), (b), and (c) are STM images of Co nanoclusters grown on the $\beta-\mathrm{Si}_{3} \mathrm{~N}_{4}(0001)$ ultrathin film $[\sim 1.2 \mathrm{~nm}$ in thickness, $p$-Si(111) substrate] at room temperature with different Co coverages. The scanning area for each image is $30 \mathrm{~nm}$ by $30 \mathrm{~nm}$. Images were obtained with the sample bias of $-4.2 \mathrm{~V}$ (filled-state images) and tunneling current of $1 \mathrm{nA}$. Below the STM images are the corresponding histograms of cluster diameter and height. not observe the occurrence of cluster coalescence even at the highest coverage $(0.36 \mathrm{ML})$. As can be seen in the STM images, the mean nearest-neighbor distance between clusters clearly decreases with increasing coverage while the mean cluster size remains the same. This is drastically different from the typical growth behavior of metallic clusters deposited on insulating substrates [7,8].

Figure 2 shows size scatter plots of supported Co (a) and (b), Fe (c), and $\mathrm{Au}(\mathrm{d})$ nanoclusters on the $\beta$ $\mathrm{Si}_{3} \mathrm{~N}_{4}(0001)$ film at different coverages. Among them, Fig. 2(a) is for room-temperature deposited Co nanoclusters and Fig. 2(b) is for comparison between as-deposited and thermally annealed Co nanoclusters, both of them showing clear linear correlation between the cluster height and diameter. This can be interpreted as a uniform cluster shape with a fixed proportion. Thus, we can model Co clusters with a constant spherical-cap shape. From the atomically resolved image on the surface reconstruction of the exposed $\mathrm{Si}_{3} \mathrm{~N}_{4}$ support, we also know that the growth is in the island growth mode (Volmer-Weber growth). Besides the Co deposition, we have also confirmed the same self-limiting growth phenomenon for supported Fe nanoclusters. The mean cluster diameter of Fe is slightly larger $(\sim 2.0 \mathrm{~nm})$ and the cluster height still distributes between 0.2 to $0.6 \mathrm{~nm}$. As shown in Fig. 2(c), nearly all the Fe nanoclusters (total number: 3264) deposited at four different coverages distribute in a strikingly narrow size window. In contrast to $\mathrm{Co}$ and $\mathrm{Fe}$ nanoclusters, $\mathrm{Au}$ nanoclusters do not exhibit the phenomenon of self-limiting size distribution and behave as predicted in the classical nucleation and growth model. This indicates that surface energy might play a unique role in such phenomena. From the size distributions of $h$ and $d$, we can determine the distribution of atom numbers in the Co clusters because of the following relationship:

$$
V=\frac{\pi}{8} d^{2} h+\frac{\pi}{6} h^{3}
$$

where $V$ is the volume of the cluster. The number of atoms in each cluster can be determined from the cluster volume $V$ and the atomic density of $\mathrm{Co}\left(\rho_{\mathrm{Co}}=9.10 \times\right.$ $10^{22}$ atoms $/ \mathrm{cm}^{3}$ ).

It is well known that cluster nucleation and diffusion often depends on the intrinsic or extrinsic surface defects. For example, previous experimental studies have revealed that supported Co nanoclusters of biatomic-layer height
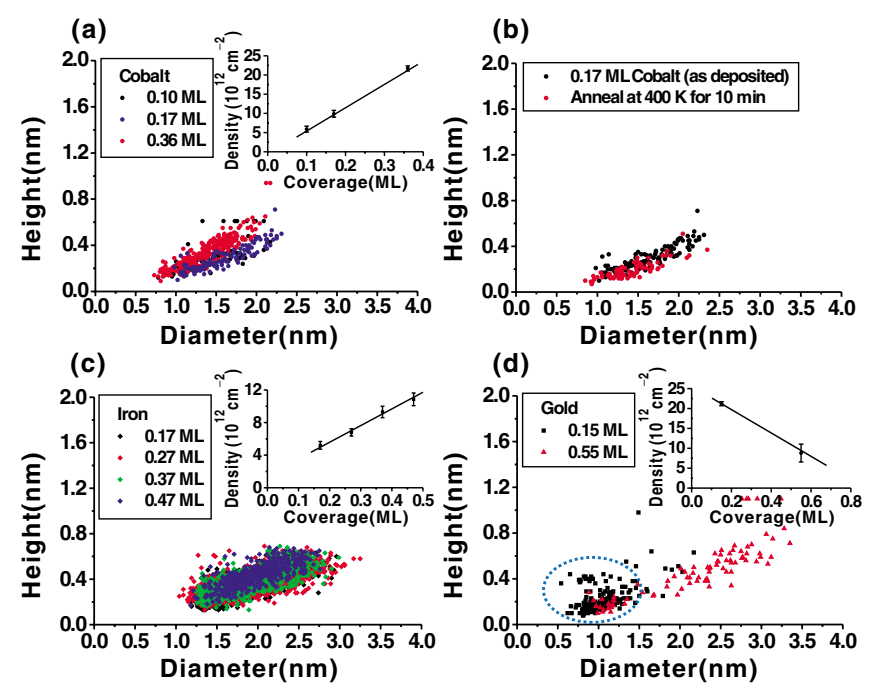

FIG. 2 (color). Scatter plots of the diameter and height of supported $\mathrm{Co}[(\mathrm{a}),(\mathrm{b}) 615$ Co clusters in (a)] Fe [(c) 3264 $\mathrm{Fe}$ clusters], and $\mathrm{Au}$ [(d) $269 \mathrm{Au}$ clusters] nanoclusters on $\beta-\mathrm{Si}_{3} \mathrm{~N}_{4}(0001)$ at different coverages. Insets in (a) and (c) show the linear relationship between the areal densities of $\mathrm{Co}$ and $\mathrm{Fe}$ nanoclusters and the deposition coverage. For $\mathrm{Au}$ nanoclusters deposited at low coverage, $95 \%$ of clusters locate within the dotted circle (blue). At high coverage, the size distribution becomes significantly wider and cluster coalescence is evident. (For $\mathrm{Co}, \mathrm{Fe}$, and $\mathrm{Au}$ deposition, $1 \mathrm{ML} \equiv 1.83 \times 10^{15}, 1.877 \times 10^{15}$, and $1.38 \times$ $10^{15}$ atoms $/ \mathrm{cm}^{2}$, respectively). 
are self-assembled on the $(23 \times \sqrt{3})$ reconstructed (socalled herringbone structure) $\mathrm{Au}(111)$ surfaces $[9,10]$. In this case, ordered two-dimensional array of Co islands, deposited at submonolayer coverage, preferentially nucleates on the "elbows" of the long-range herringbone (an ordered zigzag pattern) reconstruction of $\mathrm{Au}(111)$. Therefore, the dislocation density located at the "elbow" sites determines the areal density of the Co nanoclusters, which is independent of the Co deposition coverage. With increasing coverage, the average Co cluster size would increase upon further aggregation and finally clusters coalesce. In our experiments, we can clearly rule out the possibility of defect-mediated nucleation since the nucleation island density linearly proportions to the Co deposition coverage [see the inset of Fig. 2(a)] and the average cluster size remains the same, completely independent of the Co coverage. These results also differentiate this case from the nucleation and growth behavior of common supported cluster systems, where a linear proportionality between the cluster density and adatom coverage only is valid in the beginning of nucleation stage $(\ll 0.1 \mathrm{ML})$ and the variation of the mean cluster diameter with deposition coverage before the coalescence regime can be typically expressed by a power law relationship [11]. Furthermore, we found no correlation between the surface structures (reconstruction, step, kink, defect, etc.) and the formation of the clusters. This is very different from previous studies of forming monodispersed and ordered metal nanoclusters utilizing various surface structures [12-15].

We have also examined the thermal stability of the Co nanoclusters supported on the single-crystal $\mathrm{Si}_{3} \mathrm{~N}_{4}$ film against cluster aggregation and/or coalescence. In Fig. 2(b), we show the result of the size distribution of Co nanoclusters after the sample was annealed in situ at $400 \mathrm{~K}$ for $10 \mathrm{~min}$. Although the density of the Co nanoclusters decreases upon annealing (not shown), the cluster size and shape display a high degree of thermal stability. We have found that the decreasing density of Co clusters actually results from the thermal decomposition of the Co clusters and the decomposed Co atoms decorates at the surface step edges or are incorporated into the partially nitridated regions of the Si substrate. This result demonstrates that the observed phenomenon of self-limiting size distribution cannot be explained by the typically observed Ostwald coarsening [16] because of the absence of ripening predicted in the classical nucleation and growth model. Furthermore, clustercluster dipole interactions do not appear to be important for this case because no correlation between the average cluster size and the mean intercluster (nearest neighbors) distance is observed.

We propose that this novel nucleation behavior is actually an interplay between drastically increased surfaceto-volume ratio and quantum size effect of supported nanoclusters in the ultrasmall size regime. For standard
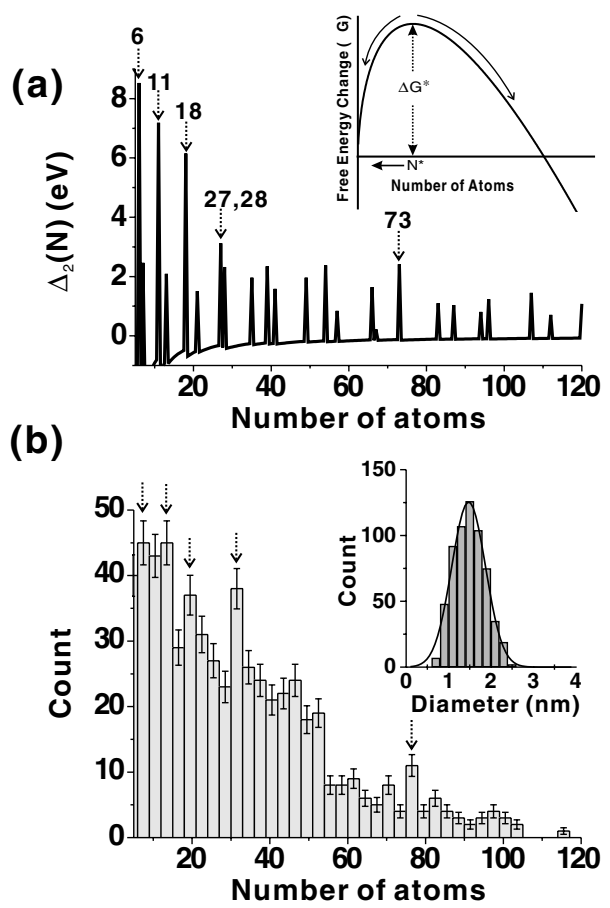

FIG. 3. (a) Calculated discrete second derivative of electronic energy $\left[\Delta_{2}(N)\right]$ vs the atomic number of Co cluster in a sequence using a hemispherical cluster geometry and infinite square-well potential. The inset in (a) shows the relation between the surface free energy $\Delta G$ and the atomic number of Co cluster. Only the local energy minima at sizes $N<N^{*}$ are relevant. (b) Abundance spectrum of Co nanoclusters obtained from three STM images shown in Figs. 1(a)-1(c). Statistical error bars are included in the histogram. The inset in (b) shows the original histogram of cluster diameter.

classical nucleation, the effect of drastically increased surface-to-volume ratio is that nucleated clusters with sizes less than the critical atom number $N^{*}$ (so-called embryo nuclei) tends to decay because their free energies would increase during growth of these nuclei, while the nuclei with sizes larger than $N^{*}$ are stable and could continue to grow since their total free energies would decrease [see the inset of Fig. 3(a)]. Here, both the nucleation barrier $\left(\Delta G^{*}\right)$ and $N^{*}$ are functions of surface (and interface) free energies and supersaturation ratio of deposition flux [7]. For metals with relatively small surface energies, the nucleation behavior is predominantly classical since the nucleation barrier is low and the size window (determined by $N^{*}$ ) for quantum nucleation is small. For Co or Fe deposition with a large cluster surface energy, the nucleation barrier is very high and the size window is also large under similar deposition conditions. Thus, nucleation can occur at certain magic sizes (less than $N^{*}$ ), resulting from the electronic shell effect of quantum confined electrons in the clusters. This is similar to previous studies on two-dimensional and one-dimensional systems (films and wires) [17-21]. To understand this phenomenon better, we use the 
hemispheric quantum dot geometry to model the confinement of valence electrons in a Co nanocluster, in which an infinite wall energy barrier confines the valence electrons of Co atoms (two $4 s$ electrons per Co atom). The reason for choosing the hemisphere shape instead of the more realistic cap shape is that the magic filling numbers of the electronic shell structure are identical for both kinds of confinement geometry [22]. And, the magic filling numbers are insensitive to the exact form of the barrier potential [4]. Therefore, the model we choose here is the simplest one to obtain the magic filling numbers because the energy eigenvalues and the corresponding degeneracies of the confined electrons in the hemisphere dot are exactly solvable. From the calculated energy spectrum, we can determine the total electronic energy $E_{e}(N)$ as a function of the atom number $N$ in the dot. The stability of each $N$-atom cluster can then be predicted by the discrete second derivative of $E_{e}(N)$, which is defined as

$$
\Delta_{2}(N) \equiv E_{e}(N+1)+E_{e}(N-1)-2 E_{e}(N) .
$$

Here, a large positive $\Delta_{2}(N)$ corresponds to a stable $\mathrm{N}$-atom cluster. Figure 3(a) displays the calculated result of $\Delta_{2}(N)$ versus $N$ for the hemispheric Co quantum dot and Fig. 3(b) is the experimental abundance spectrum of Co nanoclusters obtained from results for all three Co coverages shown in Fig. 1 [we use Eq. (1) and the known atomic density of Co to calculate total atom number in each cluster]. The experimental abundance spectrum agrees quite well (especially for $N<30$ ) with the prediction of this simple model. This demonstrates that the local energy minima of the electronic shell structure can provide several metastable sizes (smaller than the critical cluster size) for Co nucleation under appropriate growth conditions. It is interesting to note that the surface free energy for Au is much lower than that of Co. Therefore, the phenomenon self-limiting size distribution was not observed for the Au case. Although we believe that the quantum size effect plays an important role in the observed size-limiting nanocluster nucleation, further theoretical studies are necessary for a comprehensive understanding of these phenomena.

In summary, we report a very unusual clustering phenomenon of Co adatoms nucleated on the $\beta-\mathrm{Si}_{3} \mathrm{~N}_{4}(0001)$ ultrathin film. Besides the present examples, we propose that this method can become a general approach for preparing monodispersed $(N<100)$ metallic clusters on a supporting substrate, utilizing the following key concepts: (i) Deposited material has a large surface free energy (such as transition metals, $\mathrm{Fe}, \mathrm{Co}$, Ni, etc.). (ii) An insulator buffer film is formed between the substrate and the deposited material with excellent barrier properties for chemical intermixing and electronic transfer. (iii) Growth conditions are chosen such that the cluster formation barrier is high and the existence of local energy minima at certain magic sizes, which are smaller than the critical cluster size.

We acknowledge Dr. Cheng-Hung Chang and Professor Chung-Yu Mou for helpful discussions. This work was supported by the National Science Council in Taiwan and the MOE Program for Promoting Academic Excellence of Universities.

*Email address: gwo@ phys.nthu.edu.tw

${ }^{\dagger}$ Email address: mtlin@ phys.ntu.edu.tw

[1] R. Kubo, J. Phys. Soc. Jpn. 17, 975 (1962); W. P. Halperin, Rev. Mod. Phys. 58, 533 (1986).

[2] Metal Clusters at Surfaces: Structure, Quantum Properties, Physical Chemistry, edited by K.-H. MeiwesBroer (Springer, Berlin, 2000).

[3] C. T. Campbell, Surf. Sci. Rep. 27, 1 (1997); M. Bäumer and H.-J. Freund, Prog. Surf. Sci. 61, 127 (1999).

[4] W. D. Knight et al., Phys. Rev. Lett. 52, 2141 (1984); W. A. de Heer, Rev. Mod. Phys. 65, 611 (1993).

[5] H. Ahn, C.-L. Wu, S. Gwo, C. M. Wei, and Y. C. Chou, Phys. Rev. Lett. 86, 2818 (2001).

[6] C.-L. Wu, J.-L. Hsieh, H.-D. Hsueh, and S. Gwo, Phys. Rev. B 65, 045309 (2001).

[7] B. Lewis and J. C. Anderson, Nucleation and Growth of Thin Films (Academic Press, London, 1978); J. A. Veneable, G. D. T. Spiller, and M. Hanbücker, Rep. Prog. Phys. 47, 399 (1984).

[8] J. Carrey, J.-L. Maurice, F. Petroff, and A. Vaurès, Phys. Rev. Lett. 86, 4600 (2001); J.-L. Maurice et al., Philos. Mag. A 79, 2921 (1999).

[9] B. Voigtländer, G. Meyer, and N. M. Amer, Phys. Rev. B 44, 10354 (1991).

[10] O. Fruchart, M. Klaua, J. Barthel, and J. Kirschner, Phys. Rev. Lett. 83, 2769 (1999).

[11] C. R. Henry, Surf. Sci. Rep. 31, 235 (1998); C. R. Henry and M. Meunier, Vacuum 50, 157 (1998).

[12] H. Brune, M. Giovannini, K. Bromann, and K. Kern, Nature (London) 394, 451 (1998).

[13] M. Y. Lai and Y. L. Wang, Phys. Rev. Lett. 81, 164 (1998).

[14] M. Y. Lai and Y. L. Wang, Phys. Rev. B 64, 241404(R) (2001).

[15] J.-L. Li et al., Phys. Rev. Lett. 88, 066101 (2002).

[16] H. Röder, E. Hahn, H. Brune, J.-P. Bucher, and K. Kern, Nature (London) 366, 141 (1993).

[17] A. R. Smith, K.-J. Chao, Q. Niu, and C. K. Shih, Science 273, 226 (1996).

[18] Z. Zhang, Q. Niu, and C. K. Shih, Phys. Rev. Lett. 80, 5381 (1998).

[19] W. B. Su et al., Phys. Rev. Lett. 86, 5116 (2001).

[20] D.-A. Luh, T. Miller, J. J. Paggel, M. Y. Chou, and T.-C. Chiang, Science 292, 1131 (2001).

[21] A. I. Yanson, I. K. Yanson, and J. M. van Ruitenbeek, Nature (London) 400, 144 (1999).

[22] C.-H. Chang, Phys. Rev. E 67, 046201 (2003). 